\title{
MASSIVE DUST DISKS SURROUNDING HERBIG Ae/Be STARS
}

\author{
G. Sandell \\ Joint Astronomy Centre, Hilo, Hawaii, USA
}

I present preliminary results from a small $\mathrm{mm} /$ submm continuum survey, mostly photometry $(1.1 \mathrm{~mm}, 800 \mu \mathrm{m}, 450 \mu \mathrm{m}$ and $350 \mu \mathrm{m})$, but also some mapping at $800 \mu \mathrm{m}$ of Herbig Ae/Be stars and a few peculiar stars. These observations were commenced on the $15 \mathrm{~m}$ JCMT submillimetre telescope on Mauna Kea, Hawaii.

The sample included the following stars: HR 5999, AB Aur, R Mon, $\mathrm{LkH}_{\alpha}$ 198, $\mathrm{LKH}_{\alpha}$ 234, BD $+40^{\circ} 4124$, HD 200775, MCW 1080, R CrA, TY CrA, PV Cep, V 645 Cyg, and MWC 349. All stars were detected except $H R$ 5999. In the $R$ Corona Australis region it is difficult to separate the stars from the extended strong emission of the surrounding dust cloud.

If I restrict myself to stars, which have been observed at more than two wavelenghts, and for which the emission is sufficiently compact, I find that the apparent $\beta$-index is $\sim 1$, i.e. less extreme than for T Tauri stars (Weintraub et al., 1989, Ap.J. Lett (in press)) where $\beta<1$. The total masses (gas and dust), deduced form the observed continuum emission, range from a few $0.1 M_{\odot}$ to $8 M_{\odot}$ for the most extreme case $\left(\mathrm{LkH}_{\alpha} 234\right)$. Although the emission is unresolved, I argue that the emission must originate from asymmetric disk like structures surrounding the stars, because if the matter was in a shell, the stars would not be visible. Only one star, $\mathrm{LkH}_{\alpha}$ 198, appears resolved and disk like, while $\mathrm{LkH}_{\alpha} 234$, which itself is unresolved, is surrounded by a large disk perpendicular to the outflow. MCW 349, appears to be associated with very little dust, most of the emission we see is probably due to free-free emission.

\section{NEW YOUNG OBJECTS FROM THE IRAS POINT SOURCE CATALOGUE}

\author{
A. Manchado ${ }^{1}$, P. García Lario ${ }^{1}$, K. C. Sahu ${ }^{2}$, S.R. Pottasch ${ }^{2}$ \\ ${ }^{1}$ Instituto de Astrofísica de Canarias, La Laguna, Tenerife, Spain \\ ${ }^{2}$ Kapteyn Astronomical Institute, Groningen, The Netherlands
}

Three new objects have been found, when carrying out a survey of IRAS sources with colours like planetary nebulae, via low resolution spectroscopy. IRAS 14592-6311 and IRAS 07173-1733 are bright cometary nebulae, showing metal line emission very similar to V1331 Cyg $\left(\mathrm{LkH}_{\alpha} 120\right)$, which is the prototype of a certain number of T Tauri stars. They present strong P-Cygni profiles and some other peculiar characteristics. IRAS 14592-6311 is associated with a molecular cloud at a distance of $2.9 \mathrm{kpc}$, while IRAS 07173-1733 is located at the edge of the dark cloud KHAV 201. In the case of IRAS 05506+2414, it presents a bipolar structure with Herbig-Haro emission characteristics in one of the lobes, produces by shocked gas, and is located near Orion. 